Structural properties, crystal quality and growth modes of MOCVD-grown AIN with TMAI pretreatment of sapphire substrate

This content has been downloaded from IOPscience. Please scroll down to see the full text.

Download details:

IP Address: 109.171.137.210

This content was downloaded on 13/08/2017 at 08:00

Manuscript version: Accepted Manuscript

Sun et al

To cite this article before publication: Sun et al, 2017, J. Phys. D: Appl. Phys., at press: https://doi.org/10.1088/1361-6463/aa8503

This Accepted Manuscript is: (C) 2017 IOP Publishing Ltd

During the embargo period (the 12 month period from the publication of the Version of Record of this article), the Accepted Manuscript is fully protected by copyright and cannot be reused or reposted elsewhere.

As the Version of Record of this article is going to be / has been published on a subscription basis, this Accepted Manuscript is available for reuse under a CC BY-NC-ND 3.0 licence after the 12 month embargo period.

After the embargo period, everyone is permitted to copy and redistribute this article for non-commercial purposes only, provided that they adhere to all the terms of the licence https://creativecommons.org/licences/by-nc-nd/3.0

Although reasonable endeavours have been taken to obtain all necessary permissions from third parties to include their copyrighted content within this article, their full citation and copyright line may not be present in this Accepted Manuscript version. Before using any content from this article, please refer to the Version of Record on IOPscience once published for full citation and copyright details, as permission will likely be required. All third party content is fully copyright protected, unless specifically stated otherwise in the figure caption in the Version of Record.

When available, you can view the Version of Record for this article at: http://iopscience.iop.org/article/10.1088/1361-6463/aa8503 


\title{
Structural properties, crystal quality and growth modes of MOCVD- grown AIN with TMAl pretreatment of sapphire substrate
}

\section{Haiding Sun ${ }^{1}$, Feng $\mathrm{Wu}^{1}$, T. M. Al tahtamouni ${ }^{2}$, Nasir Alfaraj ${ }^{1}$, Kuang-Hui $\mathrm{Li}^{1}$, Theeradetch Detchprohm ${ }^{3}$, Russell D. Dupuis ${ }^{3}$, Xiaohang Li $^{1}$}

\author{
${ }^{1}$ King Abdullah University of Science and Technology (KAUST), Advanced Semiconductor Laboratory, Thuwal, 23955-6900, \\ Saudi Arabia \\ ${ }^{2}$ Materials Science and Technology Program, College of Arts and Sciences, Qatar University, Doha 2713, Qatar \\ ${ }^{3}$ Center for Compound Semiconductors and School of Electrical and Computer Engineering, Georgia Institute of Technology, \\ Atlanta, Georgia 30332, USA
}

Email: xiaohang.li@kaust.edu.sa

\section{Abstract}

The growth of high quality AlN epitaxial films relies on precise control of the initial growth stages. In this work, we examined the influence of the trimethylaluminum (TMAl) pretreatment of sapphire substrates on the structural properties, crystal quality and growth modes of heteroepitaxial AlN films on (0001) sapphire substrates. Without the pretreatment, the AIN films nucleated on the smooth surface but exhibited mixed crystallographic Al- (N-) polarity, resulting in rough $\mathrm{AlN}$ film surfaces. With increasing the pretreatment time from 1 to $5 \mathrm{~s}$, the N-polarity started to be impeded. However, small islands were formed on sapphire surface due to the decompostion of TMAl. As a result, small voids became noticeable at the nucleation layer (NL) because the growth started as quasi three-dimensional (3D) but transformed to 2D mode as the film grew thicker and got coalesced, leading to smoother and Al-polar films. On the other hand, longer pretreatment time of $40 \mathrm{~s}$ formed large 3D islands on sapphire, and thus initiated a 3D-growth mode of the AlN film, generating Al-polar AIN nanocolumns with different facets, which resulted into rougher film surfaces. The epitaxial growth modes and their correlation with the AIN film crystal quality under different TMAl pretreatments are also discussed.

Keywords: TMAl pretreatment, AlN film, polarity, crystal quality, growth mode

\section{Introduction}

As members of the III-nitrides family, $\mathrm{Al}_{\mathrm{x}} \mathrm{Ga}_{1-\mathrm{x}} \mathrm{N}$ alloys have become a key material for deep ultraviolet (DUV) optoelectronic devices [1-5]. Even though bulk AIN substrates have been developed and are suitable for device applications, they are still expensive and only available in small sizes. Recently, the development of AIN films on silicon $(\mathrm{Si})$ substrates showed the potential for realizing UV devices [6]. However, the crystal quality of AlN on Si was relatively low due to the large lattice and thermal mismatches between AIN and Si. Unlike sapphire, $\mathrm{Si}$ is also UV-absorptive. Thus, the growth of AlGaN-based devices is often carried out on AIN film grown on sapphire substrate. Extensive studies have been carried out on the growth optimization of the AIN films on sapphire. In particular, the initial growth conditions including hydrogen $\left(\mathrm{H}_{2}\right)$ annealing [7], growth temperatures [8, 9], thickness control of the nucleation layers (NLs) [10, 11], and the III/V ratio [12] have shown significant impacts on the surface morphology, polarity and crystal quality of the MOCVD-grown AIN films. Inappropriate growth temperatures and thicknesses of the NL could lead to generating mixed polarities and inversion domains in $\mathrm{AlN}$ films.
Moreover, it has been reported that the pretreatment of sapphire substrates either by ammonia $\left(\mathrm{NH}_{3}\right)$ or trimethylaluminum (TMAl) is strongly correlated with the surface morphology [13,14], polarity [15], and crystal quality [16]. Researchers demonstrated that atomically smooth Al-polar AlN films were obtained without nitridation [16]. Other research group have shown that mixed polarity by pre-growth nitridation of the sapphire substrate results in rough film surfaces because of the different growth rates of N-polar and Al-polar AlN films $[8,17]$. Theoretically, from ab initio studies, the nitridation is described as the N-rich limit of growth, with sapphire as the source of $\mathrm{Al}$, and may result in different polarities of AlN films [18]. Experimentally, it was proposed that the formation of $\mathrm{Al}_{\mathrm{x}} \mathrm{O}_{\mathrm{y}} \mathrm{N}_{\mathrm{Z}}$ is what leads to the rough surfaces and the mixed polarity of the AIN films [19, 20]. Recently, Mohn et al. achieved Al-polar AlN films on a nitridated sapphire substrate by carefully controlling the $\mathrm{Al}_{\mathrm{x}} \mathrm{O}_{\mathrm{y}} \mathrm{N}_{\mathrm{Z}}$ intermediate layer followed by a high-temperature annealing process [13].

Nitridation is recognized as a crucial step and thus has been investigated extensively for the sake of achieving desirable AlN or GaN films grown by MOCVD, yet not much attention was paid to understanding the crystallographic polarity and growth evolution of nitride films achieved through TMAl pretreatment (i.e. 
alumination). The topic of defective and rough-surface AlN/sapphire templates resulting from the TMAl pretreatment has been addressed before [21, 22]. Reentila et al. have argued that neither nitridation nor alumination gave the best structural quality [16]. In contrary, studies have shown that better AlN and GaN crystal quality can be achieved after pretreatment using TMAl [23, 24]. Recently, we revealed that it was the carbon presence at the AlN/sapphire interface after TMAl pretreatment that changed the polarity of AlN film. However, the crystal
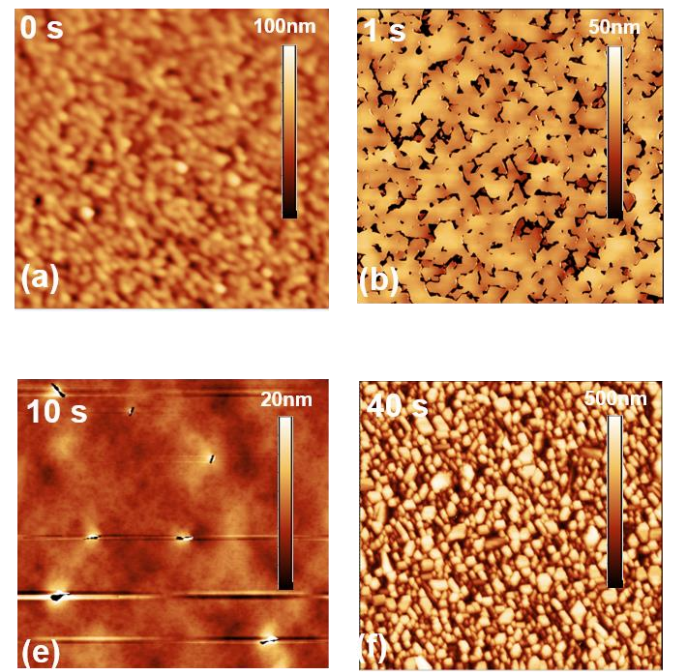

quality of AlN film after the pretreatment was not analyzed [25].

In this study, we present a comprehensive investigation of the structural properties, including surface morphology, polarity, and crystal quality of AlN templates under different TMAl pretreatment durations of sapphire substrates. We also analyzed the correlation between the growth modes induced by different pretreatment and the crystal quality of AlN templates.

\section{Experimental details}
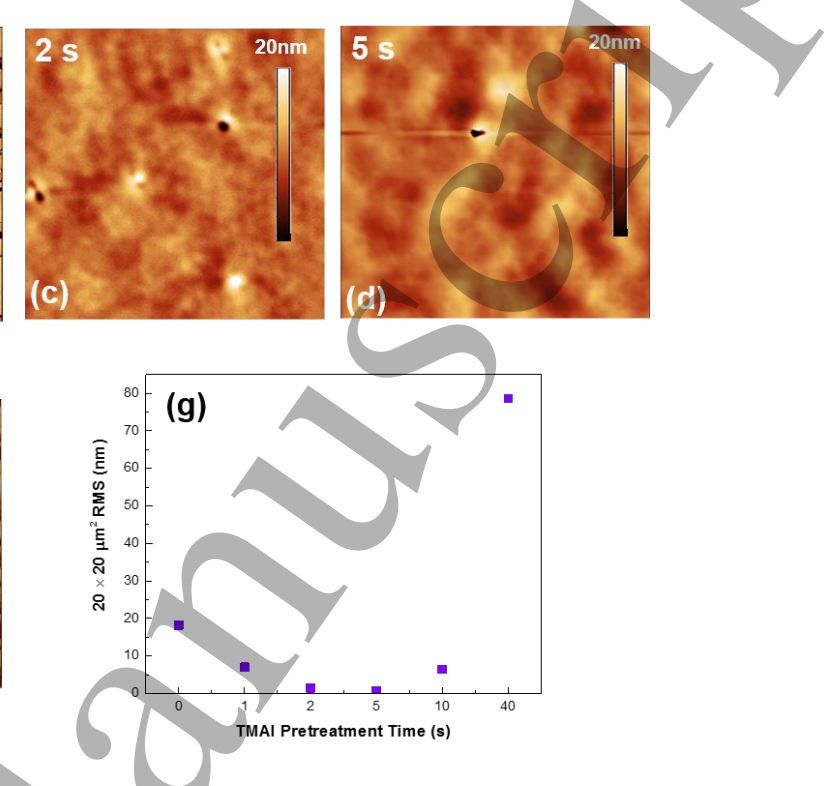

Figure 1. (a) - (f) show the $20 \times 20 \mu \mathrm{m}^{2}$ AFM images of the surface morphology of $3 \mu \mathrm{m} \mathrm{AlN} /$ sapphire layers grown with pretreatment times of $0,1,2,5,10$ and $40 \mathrm{~s}$, respectively. (g) corresponds to the extracted RMS roughness value from AFM images with different pretreatment times.

The AlN heteroepitaxial layers were grown on a c-plane (0001) sapphire substrate using an AIXTRON ClosedCoupled Showerhead metalorganic chemical vapor deposition (MOCVD) system which has a base pressure of $\sim 1.5 \times 10^{-2}$ Torr. TMAl and $\mathrm{NH}_{3}$ were used as precursors and $\mathrm{H}_{2}$ as a carrier gas. The sapphire substrates were taken from the original package and loaded into the reactor immediately without solvent cleaning. The AlN growth processes comprised four steps. First, the susceptor and sapphire substrates were baked at $1100^{\circ} \mathrm{C}$ for $5 \mathrm{~min}$ in the $\mathrm{H}_{2}$ ambient environment. Second, a preflow of TMAl was introduced to the substrate with pretreatment times of 0,1 , $2,5,10$, and $40 \mathrm{~s}$ at a same flow rate of $1.72 \times 10^{-5} \mathrm{~mol} . / \mathrm{min}$ with a chamber pressure of 85 mbar during the pretreatment. Then the growth started with a $15 \mathrm{~nm}$ AlN $\mathrm{NL}$ at a temperature of $\sim 900^{\circ} \mathrm{C}$. Last, a $\sim 3 \mu \mathrm{m}$ AlN layer was grown at $\sim 1100^{\circ} \mathrm{C}$. Except for the pretreatment times of the TMAl, the other growth parameters were the same for all samples. The reactor pressure was 85 mbar during the growth. Besides AlN films, we also prepared two samples with 5 and $40 \mathrm{~s}$ TMAl pretreatment (hereafter TMAl-only samples) under the identical MOCVD condition, without the subsequent AlN growth. The surface morphology and roughness of the AlN films and TMAl-only samples were examined by atomic force microscopy (AFM). Microstructures were revealed via high-angle annular dark-field scanning transmission electron mícroscopy (HAADF-STEM) at an acceleration voltage of $300 \mathrm{kV}$ in an FEI Titan Probe microscope system. The TEM specimens were prepared by focused lon beam (FIB) using an FEI Helios SEM system with a $\mathrm{Ga}$ ion source. High resolution-X-ray diffraction (HRXRD) was performed to characterize the crystalline quality.

\section{Results and discussions}

Figure 1 shows the AFM root mean square (RMS) roughness and surface morphology of the AlN templates. The results exhibit significant difference in surface morphology. Without TMAl pretreatment, the sample exhibited a rough surface with pits. The surface morphology gradually smooths out as the pretreatment time increased from 0 to $5 \mathrm{~s}$. Small pits (threading dislocation centers) are still visible in the sample with the $5 \mathrm{~s}$ pretreatment. However, the surface became rougher again with a longer pretreatment time for the sample at 10 $\mathrm{s}$ duration. After the $40 \mathrm{~s}$ pretreatment, the sample had the roughest surface among the investigated AlN templates. The smoothest AlN film was obtained at under the $5 \mathrm{~s}$ pretreatment time with an RMS roughness of less than 1 $\mathrm{nm}$. The sample with the $40 \mathrm{~s}$ pretreatment time yielded an RMS value of $80 \mathrm{~nm}$, indicating a 3D growth condition of AlN film might be established after such long pretreatment. Thus, without and excessive pretreatment 
both led to a rough surface. With proper pretreatment duration, a smooth AlN/sapphire template is achievable.

Next, we carried out detailed TEM analysis of the three AlN/sapphire samples with pretreatment times of 0 , 5, and 40 s. Figure 2 (a) shows an overall HAADF-STEM image of the AlN/sapphire template without pretreatment. We observed many domains with inverted cone shapes embedded with bright and dark vertical stripes along the growth direction (0001). A rough surface was also observed. Meanwhile a large number of threading dislocations (TDs, in white contrast) were created at the interface but slightly diminished in density as the thickness of the AlN layer increased (fewer TDs above the white dashed line). Nevertheless, the stripes propagated from the interface of the AlN/sapphire to the surface of the AlN template and its contrast alternately changed along the in-plane direction.

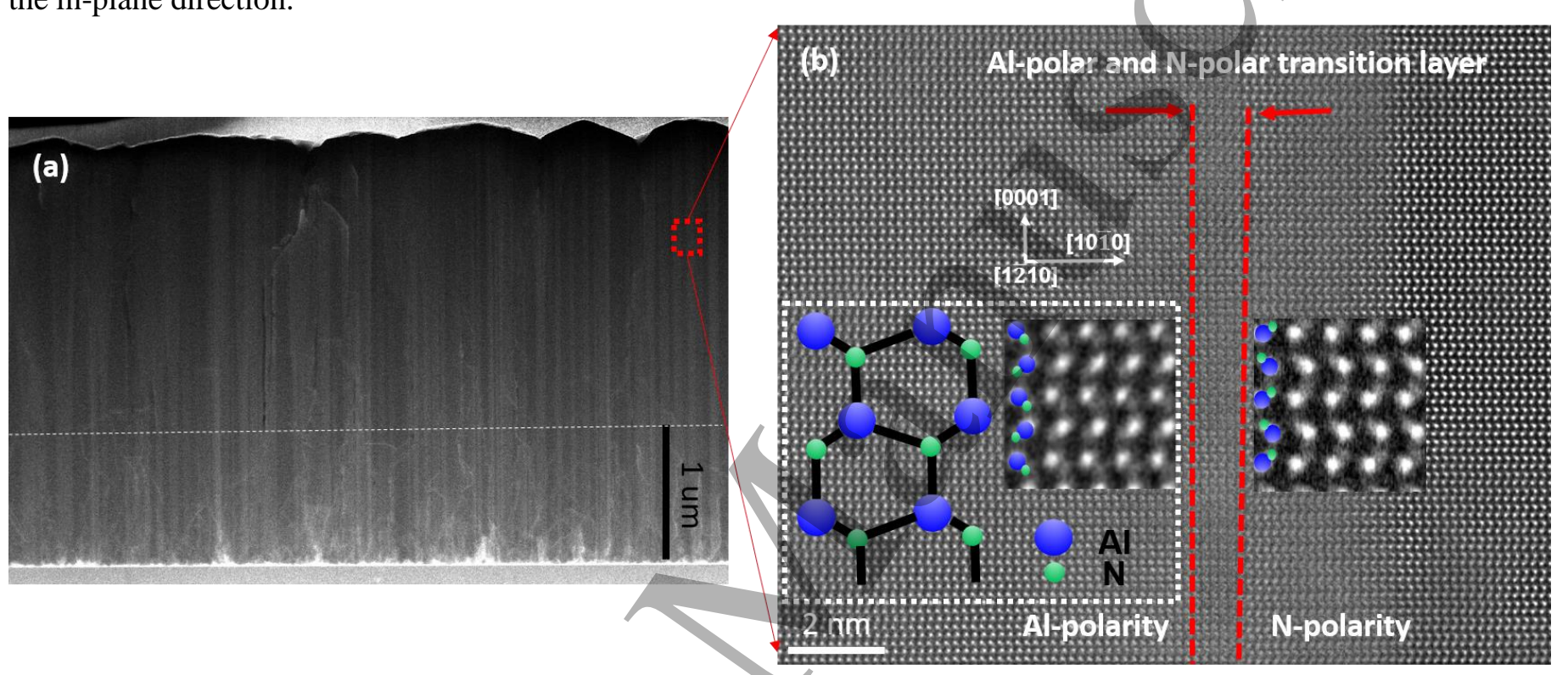

Figure 2. (a) An overall HAADF-STEM image of the AlN/sapphire template without pretreatment. (b) Observed atomic resolution image of the red dashed box in (a), including two enlarged images for two stripes and the transition layer. Inset image shows the schematic of atomic structure of Al-polar AlN film.

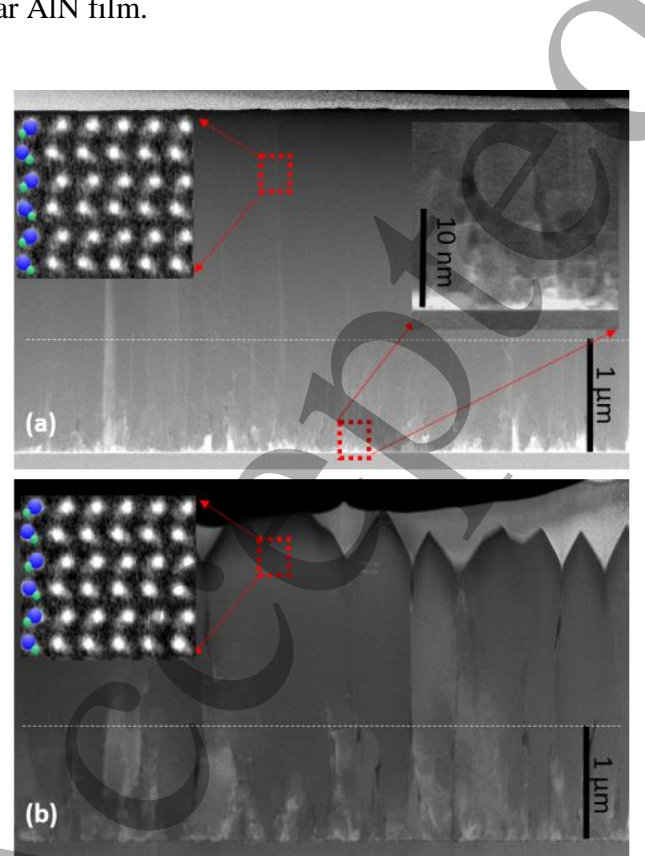

Figure 3. (a) and (b) are overall HAADF-STEM images of the AIN/sapphire template with $5 \mathrm{~s}$ and $40 \mathrm{~s}$ pretreatment respectively. The inset images in (a) are the atomic resolution image (left) and high magnification image (right), showing the polarity of the AlN template
Figure 2 (b) shows two polarities in two strips next to one another. The left stripe has Al-polarity and the right one has N-polarity, confirming the mixed polarity of the film. There is an inversion domain boundary (IDB), which is marked by the red dashed line. A few groups reported that the mixed polarity is primarily caused by the formation of an $\mathrm{Al}_{\mathrm{x}} \mathrm{O}_{\mathrm{y}} \mathrm{N}$ interlayer between the sapphire and the AlN-NL. This $\mathrm{Al}_{\mathrm{x}} \mathrm{O}_{\mathrm{y}} \mathrm{N}$ interlayer causes the polarity to be inverted to the N-polarity [26, 27]. Recent discoveries have also shown that decomposition of sapphire prior to the growth led to the simultaneous growth of $\mathrm{N}$ - and Al-polar AlN films [28]. The growth rate of Al-polar film was found to be much higher than that of N-polar films [17], thus leading to a rough surface, as shown in Figure 2 (a), that is not beneficial to subsequent film growth. and the existence of pore structures at the interface respectively. The inset image in (b) is the atomic resolution image.

After $5 \mathrm{~s}$ pretreatment, the sample exhibits a uniform and smooth surface, as shown in Figure 3 (a). Although TDs were crowded throughout the interface between the AlN layer and the sapphire, the TDs rapidly vanished once the thickness increased beyond $1 \mu \mathrm{m}$ (white dashed line) and only a few TDs propagated to the surface. Interestingly, we observed a high density of small voids in the bottom section of the AlN template. These nanoscale voids could be the result of the incomplete coalescence of the nucleation islands. Literatures proposed that a few monolayers of $\mathrm{Al}$ were deposited on the sapphire's surface $[29,30]$ and formed the nucleation islands before $\mathrm{NH}_{3}$ came into the reaction chamber, creating a quasi-threedimensional (quasi 3D) growth mode at the beginning of the growth. Later, the high-temperature AlN began to grow on these quasi-3D islands, which was followed by the islands' coalescing and finally the growth transferred to a $2 \mathrm{D}$ mechanism. The voids were suppressed and the TDs were bent and/or annihilated as the film grew thicker [31], forming a uniform and smooth film. The inset image 
shows the atomic resolution image, confirming the Alpolarity. As a result of the homogeneous and uniform Alpolarity across the wafer and lack of inversion domains, we obtained a uniform growth rate across the entire 2-inch wafer. In accordance with the AFM measurement, the result suggested that the RMS roughness value reduces after the pretreatment time increased beyond 0 to $5 \mathrm{~s}$. The improved surface morphology is mainly attributed to uniform polarity given that the proportion of Al-polarity increased by suppressing the oxygen diffusion from the sapphire after the pretreatment. With the $5 \mathrm{~s}$ pretreatment, the formation of $\mathrm{N}$-polarity regions was completely impeded [32].

Figure 3(b) shows that the overall HAADF-STEM image of the AlN/sapphire template with the $40 \mathrm{~s}$ pretreatment that exhibited the roughest surface among the investigated samples. The surface had faceted nanocolumns. Meanwhile the AlN/sapphire interface included a high density of voids and TDs. This is primarily due to excessive pretreatment of the sapphire, resulting in the formation of high density large 3D islands (domains) at NL. Thus, a 3D growth mode was immediately established after the pretreatment, creating nanocolumns with well-defined boundaries. Most of the TDs formed at the interface or diminished at the boundary, leaving less density at the top part of the nanocolumns. Similar to the other two samples, the TDs rapidly vanished as the thickness of the AlN layer increased beyond $1 \mu \mathrm{m}$ (white dashed line). The inset image confirms the Al-polarity of this film.

Next, we will address the correlation of TMAlpretreated sapphire surface with the crystal quality and the growth mechanism of AlN films.

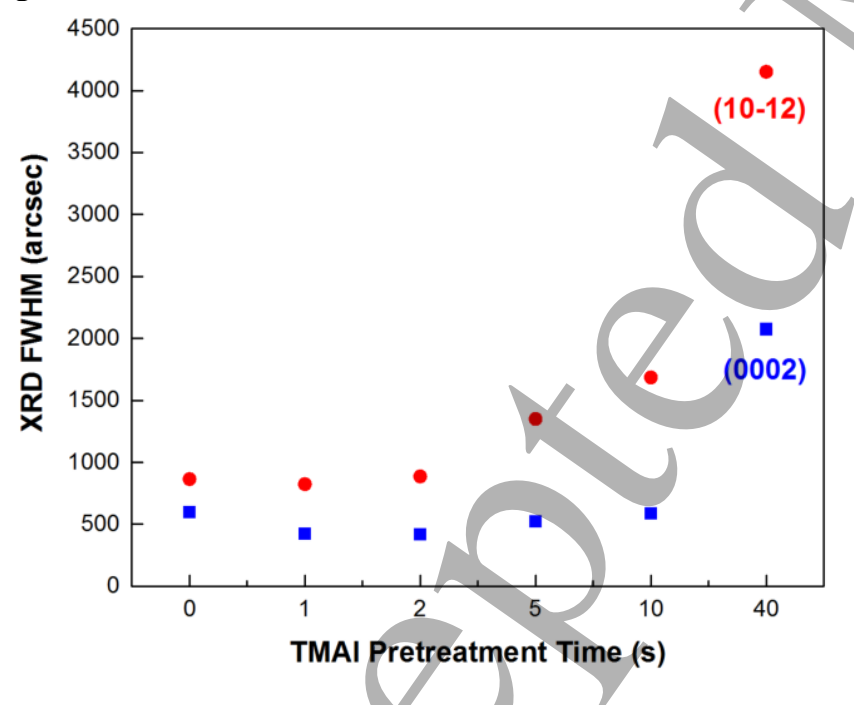

Figure 4. FWHM's of XRD (002) and (102) $\omega$-scans on the AlN templates with different pretreatment time.

The crystal quality of the AIN templates was studied by measuring the full width at half maximum (FWHM) of the XRD symmetric (002) and asymmetric (102) $\omega$-scans, as shown in Figure 4 [33-35]. Except for the sample with the 40-s pretreatment, the obtained FWHM values of XRD peaks were largely comparable with the ones reported by other groups, falling in the range of 100 500 and 800 1500 arcsec for the (002) and (102) plane, respectively [36-39]. The FWHM values of the symmetric
(002) and asymmetric (102) $\omega$-scans corresponded to and were proportional to the screw dislocation and the edge dislocation densities, respectively. It is generally noted that the screw dislocations and edge dislocations originate from the stacking faults that cause tilting and twisting grain boundaries. A close examination of Figure 4 reveals different trends of the crystalline quality with different pretreatment times. The (002) FWHM (blue curve) is nearly constant during the first $10 \mathrm{~s}$ pretreatment, indicating that our relatively lower temperature $\left(\sim 900^{\circ} \mathrm{C}\right)$ pretreatment processes did not result in a large tilt of AlN nuclei, thus the density of screw dislocation did not change too much. For the (102) FWHM/(red curve), it slightly decreases after the $2 \mathrm{~s}$ pretreatment and then increases significantly from 5 to 40 s pretreatment duration, indicating higher density of edge/dislocations. The smallest (002) and (102) FWHMs were obtained when the pretreatment time was 1 2 $\mathrm{s}$ and the (102) FWHM is more sensitive to the pretreatment time. A similar XRD trend was observed in the study of the pretreatment of sapphire using TMAl [32] for MOCVD-grown GaN templates. However, our observation is contrary to what O. Reentila et al. reported earlier [16]. They presented both FWHMs increased dramatically with the increase of TMAl exposure time, and the surface roughness of their films remained below $1 \mathrm{~nm}$, even after the $80 \mathrm{~s}$ pretreatment. Thus, we believe the discrepancy is probably due to higher growth temperature of $1500^{\circ} \mathrm{C}$ and $1000^{\circ} \mathrm{C}$ for their AlN template and AlN-NL respectively. Normally, higher growth temperatures (generally more than $1400{ }^{\circ} \mathrm{C}$ ) are favorable for the migration of $\mathrm{Al}$ adatoms, which benefits the increase of crystal quality of AlN templates [40]. Another study has shown that the roughness of the NL decreased by increasing the growth temperature of the NL $\left(T_{\mathrm{NL}}\right)$ because the diffusion length of $\mathrm{Al}$ adatoms and lateral coherence length at sapphire surface increased as $T_{\mathrm{NL}}$ was increased [41]. So the growth temperature plays such significant role in the process of AlN film by MOCVD.

Besides the variation of the crystal quality, TEM studies (Figure 2 and 3 ) revealed the drastic microstructure difference between samples underwent various pretreatment conditions. Most notably, the growth modes have undergone significant transformations as a result of changing the pretreatment times. Here we present the investigation on the surface morphology of the TMAlonly samples using AFM prior to the AlN epitaxy, as shown in Figure 5. Without pretreatment, the sapphire surface was extremely smooth, with a measured RMS value of $0.2 \mathrm{~nm}$. The cross-section profile across the scanned surface shows less than $1 \mathrm{~nm}$ in height. However, after 5 s pretreatment, we clearly observed spotty surface with a measured RMS value of $2.3 \mathrm{~nm}$, indicating a formation of rough surface with small islands on the sapphire substrate. The cross-section profile indicates an 8 - $10 \mathrm{~nm}$ in height. With $40 \mathrm{~s}$ pretreatment, irregular nanoscale 3D islands $(50-100 \mathrm{~nm}$ in horizontal dimension) on sapphire substrates were formed, with a measured RMS value of $6.3 \mathrm{~nm}$. The cross-section profile indicates a measured height ranges of $15-20 \mathrm{~nm}$. 
(a)
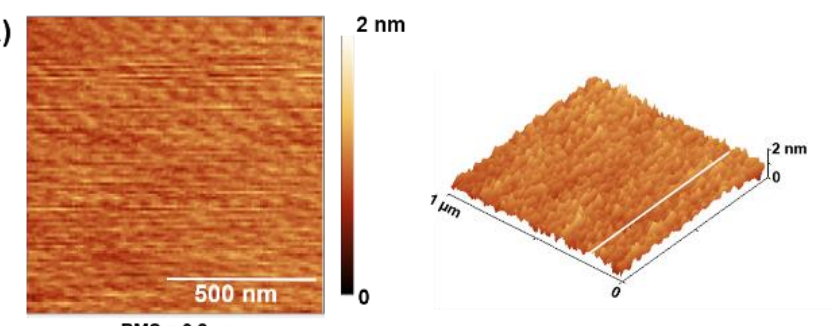

(b)

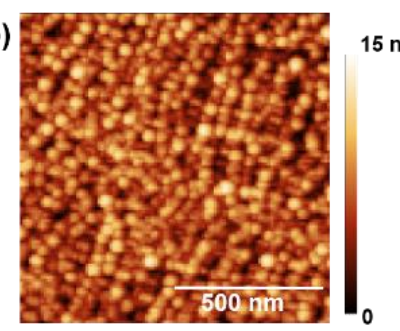

$\mathrm{RMS}=\mathbf{2 . 3} \mathrm{nm}$
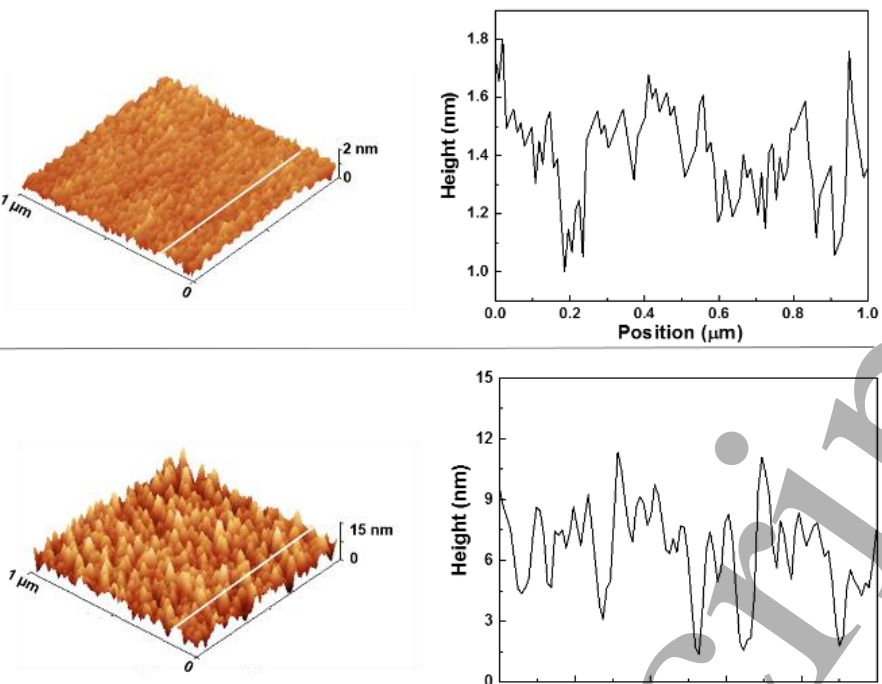

(c)
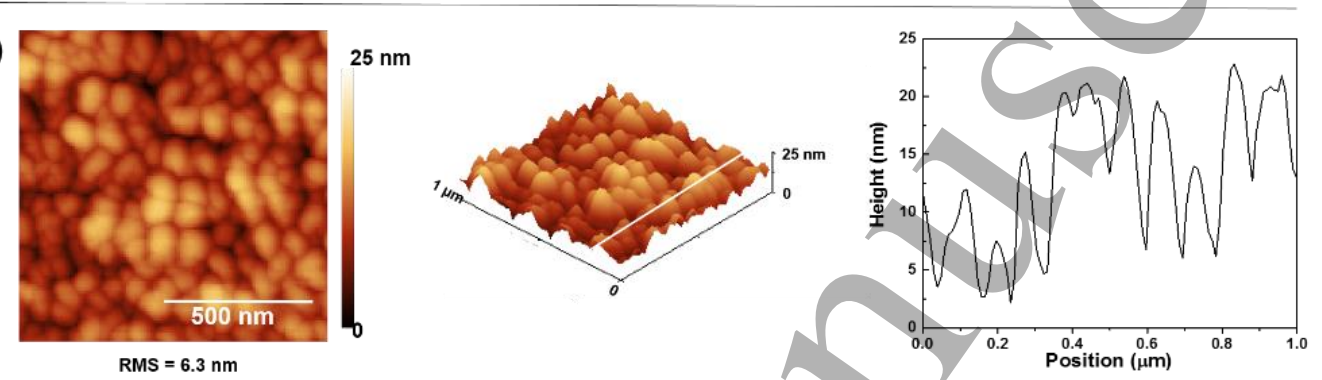

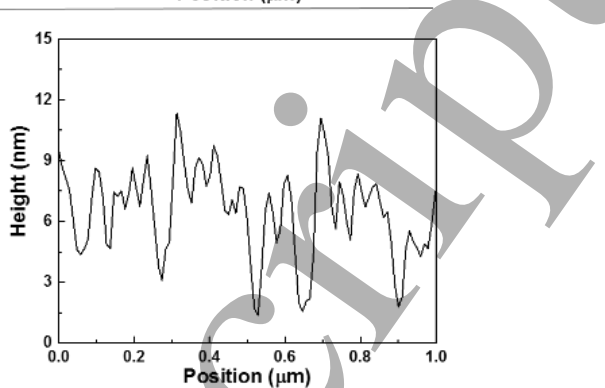

Figure 5. (a), (b) and (c) are AFM images of surface morphology of TMAl-only samples (without AlN epitaxy) with the 0,5 and $40 \mathrm{~s}$ pretreatment, respectively. The left, middle, and right are the plan-view, 3D image and cross-section profiles, respectively, corresponding to the marked line in the 3D image.

(a) Os pretreatment

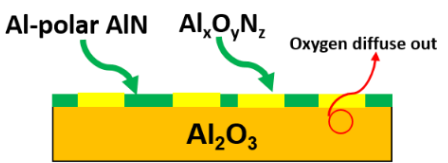

(b) 5s pretreatment

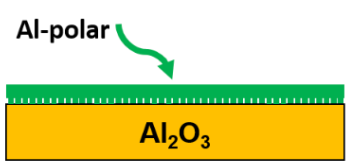

(c) $40 \mathrm{~s}$ pretreatment

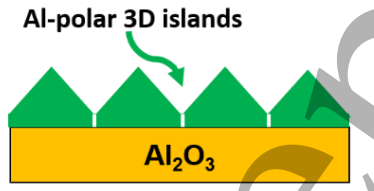

Figure 6. Schematic illustration of the growth modes, the evolution of the AlN templates with (a) $0 \mathrm{~s}$, (b) $5 \mathrm{~s}$, and (c) $40 \mathrm{~s}$ pretreatment

Based on the observation of the structural characteristic of AlN films and TMAl-only samples above, here we propose the growth mechanism under 0,5 and 40 s pretreatment. Figure 6(a) shows the creation of rough AlN films without pretreatment. Although the AlN started nucleation on smooth sapphire, because of the formation of $\mathrm{Al}_{\mathrm{x}} \mathrm{O}_{\mathrm{y}} \mathrm{N}_{\mathrm{z}}$ in the first few monolayers of $\mathrm{AlN}$ growth $[13,17], \mathrm{Al}$-polarity was partially transformed to N-polarity and this has led to the mixed polarity of AlN film, thus created a rough surface. However, after the TMAl pretreatment, the process not only helped establish the Al-polar growth condition but also create a slightly roughened surface due to the TMAl decomposition, confirmed by AFM in Figure 5(b) [42]. The residuals of decomposed TMAl formed a cluster of small islands, thus â quasi-3D growth mode was introduced. The formation of small islands, which was accounted for inducing the quasi-3D growth mode, was proved to be beneficial to create higher quality of AIN and GaN films [23, 24]. As the growth continued, these small AlN grains coalesced and the surface became smooth, which meant a 2D-growth (a layer-by-layer growth) was established in the end. This process is similar to the high-quality and smooth GaN growth on sapphire with a combination of quasi-3D and then 2D growth modes [43, 44]. Figure 6 (b) presents the schematic of such growth mechanism for the $5 \mathrm{~s}$ pretreatment. A uniform and smooth surface of AlN template was obtained. In addition to the improvement of surface morphology, an increase of the density of edge dislocations ((102) FWHMs of XRD $\omega$-scan) was observed by increasing the pretreatment time. This might be due to the increasing size of islands formed on sapphire after longer pretreatment time, as shown in Figure 5, preventing the migration of arriving $\mathrm{Al}$ and $\mathrm{N}$ atoms horizontally. These islands facilitated in generating more twisted grain boundaries while establishing the initial quasi-3D growth mode, thus a higher density of edge dislocations. Also, the larger the islands prior to the AlN epitaxy on sapphire, the thicker subsequent AlN film might be required to get fully coalesced while creating small voids in the bottom AlN layer [23]. Nevertheless, 
these islands did not contribute to the tilt of in-plane AIN nuclei, thus little impact on the density of screw dislocations after increasing the pretreatment time from 1 to $10 \mathrm{~s}$. Once the sapphire substrate is over-treated, a high density of larger 3D islands were formed on sapphire (Figure 5(c)), leading to extreme rough NL. The NL might act as a mask and further prevent Al-adatoms from diffusion to different nucleation islands, thus leading to a $3 \mathrm{D}$ growth mode throughout the subsequent template growth. The Al-polar AIN template with nanocolumns was grown on those islands which are consistent with the AFM measurements, as shown in Figure 6(c). Interestingly, Eom et al. were able to fabricate AlN 3D nanostructures using $\mathrm{KOH}$ etching by controlling the polarity of the AlN films due to the TMAl preflow [45].

\section{Conclusions}

In conclusion, we have studied the influences of the TMAl pretreatment on the structural properties, crystal quality and growth mode of AIN films on sapphire substrates by MOCVD. Due to the absence of the TMAl pretreatment, the AIN films had a mixed polarity, but after a few seconds of pretreatment, the polarity of AlN films tended to convert to Al-polarity, while the surface roughness and crystal quality were improved according to the FWHM variations of the (102) and (002) rocking curves. However, excessive pretreatment results in poorer crystal quality and a rough surface. A detailed epitaxial growth mechanism under different pretreatment time were presented. A shift of growth mode from quasi-3D to 3D was identified after increasing the pretreatment time. We conclude that pretreatment times that are too short or too long should be avoided as neither is favorable for AlN epitaxy on sapphire. These results give practical advice and guidance in changing the growth mode of AlN film to achieve desirable AlN films.

\section{Acknowledgements}

The KAUST authors would like to acknowledge the support of GCC Research Program REP/1/3189-01-01, Baseline BAS/1/1664-01-01, and Equipment BAS/1/1664-01-07. The work at QU was supported by GCC Research Program GCC-2017-007. The work at Georgia Institute of Technology was supported in part by DARPA under grant W911NF-15-1-0026 and NSF under grant DMR-1410874. RDD acknowledges the additional support of the Steve W. Chaddick Endowed Chair in Electro-Optics and Georgia Research Alliance.

\section{References}

[1] Janjua B et al. 2017 Opt. Express 251381.

[2] Janjua B et al. 2017 Nanoscale 97805.

[3] Sun H and Moustakas D T 2014 Appl. Phys. Express 7012104.

[4] Pecora E F, Sun H, Negro L D and Moustakas T D 2015 Opt. Mater. Express 5809.

[5] Sun H, Yin J, Pecora E F, Dal Negro L, Paiella R and Moustakas T D, 2017 IEEE Photon. J. DOI: 10.1109/JPHOT.2017.2716420
[6] Tran B T, Maeda N, Jo M, Inoue D, Kikitsu T and Hirayama H 2016 Sci. Rep. 635681.

[7] Kumagai Y, Akiyama K, Togashi R, Murakami H, Takeuchi M, Kinoshita T, Takada K, Aoyagi Y and Koukitu A 2007 J. Cryst. Growth 305366.

[8] Wu Y, Hanlon A, Kaeding J F, Sharma R, PFini T, Nakamura S and Speck J S 2004 Appl. Phys. Lett. 84912.

[9] Li X H et al. 2015 J. Cryst. Growth 41476.

[10] Miyagawa R, Yang S, Miyake H, Hiramatsu K, Kuwahara T, Mitsuhara M and Kuwano N 2012 Appl. Phys. Express 5025501.

[11] Li H N, Sadler T C and Parbrook P J 2013 J. Cryst. Growth 38372.

[12] Claudel A, Fellmann V, Gélard I, Coudurier N, Sauvage D, Balaji M, Blanquet E, Boichot R, Beutier G and Coindeau S 2014 Thin Solid Films $\mathbf{5 7 3} 140$.

[13] Mohn S et al.2016 Phys. Rev. Applied 5054004.

[14] Takeuchi M et al. 2007 J. Cryst. Growth 305360.

[15] Jasinski J, Liliental-Weber Z, Paduano Q S and Weyburne D W 2003 Appl. Phys. Lett. 832811.

[16] Reentila O, Brunner F, Knauer A, Mogilatenko A, Neumann W, Protzmann H, Heuken M, Kneissl M, Weyers M and Trankle G 2008 J. Cryst. Growth 3104932.

[17] Paduano Q S, Weyburne D W, Jasinski J and Weber Z L $2004 \mathrm{~J}$. Cryst. Growth 261259.

[18] Di Felice R and Northrup J E 1998 Appl. Phys. Lett. 73936.

[19] Dwikusuma F and Kuech T F 2003 J. Appl. Phys. 945656.

[20] Losurdo M, Capezzuto P, Bruno G, Namkoong G, Doolittle W A and Brown A S 2002 J. Appl. Phys. 912508.

[21] Kawaguchi K and Kuramata A 2005 Jpn. J. Appl. Phys. 44 L1400.

[22] Kueller V, Knauer A, Brunner F, Mogilatenko A, Kneissl M and Weyers M 2012 Phys. Status Solidi C 9496.

[23] Bao Q, Luo J and Zhao C 2014 Vacuum 101184.

[24] BakSS J, Mun D H, Jung K C, Park J H, Bae H J, Lee I W, Ha J S, Jeong T and Oh T S 2013 Electron. Mater. Lett. 9367.

[25] Sun H, Wu F, Park Y J, Altahtamouni T M, Li K H, Alfaraj N, Detchprohm T, Dupuis R D, Li X, 2017 Appl. Phys. Lett. 110 192106.

[26] Hussey L et al. 2014 Appl. Phys. Lett. 104032104.

[27] Wong M H, Wu F, Speck J S and Nakamura S $2010 \mathrm{~J}$. Appl. Phys. 108123710.

[28] Kumagai Y, Enatsu Y, Ishizuki M, Kubota Y, Tajima J, Nagashima T, Murakami H, Takada K and Koukitu A $2010 \mathrm{~J}$. Cryst. Growth $\mathbf{3 1 2} 2530$.

[29] Harumoto T, Sannomiya T, Matsukawa Y, Muraishi S, Shi J, Nakamura Y, Sawada H, Tanaka T, Tanishiro Y and Takayanagi K 2013 J. Appl. Phys. 113084306.

[30] Li C, Liu H and Chua S J 2015 J. Appl. Phys. 117125305.

[31] Li X H et al. 2015 Phys. Status Solidi B 2521089.

[32] Lim D H, Xu K, Arima S, Yoshikawa A and Takahashi K $2002 \mathrm{~J}$. Appl. Phys. 916461.

[33] Wang H M, Zhang J P, Chen C Q, Fareed Q, Yang J W and Khan M A, 2002 Appl. Phys. Lett. 81604.

[34] Heying B, Wu X H, Keller S, Li Y, Kapolnek D, Keller B P, DenBaars S P and Speck J S, 1996 Appl. Phys. Lett. 68643.

[35] Ayers J E 1994 J. Cryst. Growth 13571.

[36] Imura M et al 2007 J. Cryst. Growth 300136.

[37] Peng M Z et al 2008 Chin. Phys. Lett. 252265.

[38] Chen Y, Song H, Li D, Sun X, Jiang H, Li Z, Miao G, Zhang Z and Zhou Y. 2014 Mater. Lett. 11426

[39] Pantha B N, Dahal R, Nakarmi M L, Nepal N, Li J, Lin J Y, Jiang H X, Paduano Q S and Weyburne D, 2007 Appl. Phys. Lett. 90 241101.

[40] Sun X J, Li D B, Chen Y R, Song H, Jiang H, Li Z M, Miao G Q and Zhang Z W 2013 Cryst. Eng. Comm. 156066.

[41] Chen Y, Song H, Li D, Sun X, Jiang H, Li Z, Miao G, Zhang Z and Zhou Y 2014 Mater. Lett. 11426.

[42] Ambacher A, Brandt M S, Dimitrov R, Metzger T, Stutzmann M, Fischer R A, Miehr A, Bergmaier A and Dollinger G, $1996 \mathrm{~J}$. Vac. Sci. Technol. B 143532.

[43] Zhao D G, Zhu J J, Liu Z S, Zhang S M, Yang H and Jiang D S 2004 Appl. Phys. Lett. 851499.

[44] Xu Z Y, Xu F J, Wan g J M, Lu L, Yang Z J, Wang X Q and Shen B 2016 J. Cryst. Growth 450160.

[45] Eom D, Kim J, Lee K, Jeon M, Heo C, Pyeon J, and Nam O 2015 J. Nanosci. Nanotechnol. 155144. 